\title{
The Use of (AHP) GIS Extension to Determine the Optimum Locations
}

\author{
${ }^{1}$ Asma Th. Ibraheem, ${ }^{2}$ Zainab H. Mahdi and ${ }^{3}$ Thikra Najah \\ ${ }^{1}$ Department of Civil Engineering, College of Engineering, Al-Nahrain University, Baghdad, Iraq \\ ${ }^{2}$ Department of Building and Construction Technology Engineering, \\ ${ }^{3}$ Department of Surveying Technology Engineering, Middle Technical University, Baghdad, Iraq
}

\begin{abstract}
Considering everything that's occurring in the study area the (Middle Technical University) (MTU) which is located in Baghdad/Al-Zafarania it is essential to locate the optimum location for numerous facilities of the university (health Centre, mosque, cafeteria, parking) and for that reason the GIS extension (Analytical Hierarchy Process) (extAHP 2.0) is implemented in this research, the used criteria are: proximity to the existing facility, proximity to classrooms, proximity to the borders.
\end{abstract}

Key words: Extension, optimum, analytical, proximity, facilities, implemented

\section{INTRODUCTION}

The process of choosing the right location for any facility is quite difficult because the responsible authority should take many factors into consideration before making any decision (Kuo et al., 1999; Mikhailov and Tsvetinov, 2004).

Therefore, (AHP) is considered very helpful in the matter of a Multi-Criteria Decision Making (MCDM) (Harker and Vargas, 1987), since, it was initially founded by Saaty and it became one of the most popular means for determining decisions and calculating weights, (Ibraheem et al., 2015) then in 2009 ArcGIS developed an extension that calculates weights of the raster layers in the table of contents by comparing their importance in a comparison matrix (Chatterjee and Mukherjee, 2013; Akalin et al., 2016).

\section{MATERIALS AND METHODS}

Methodology of AHP extension: Reaching the goal of finding the optimum location can be done by performing these steps.

\section{Cafeteria}

Choosing set of criteria for each facility: Which are (proximity to the existing facility, proximity to classrooms, proximity to open spaces).

Generating a map for the chosen criteria: By performing these following.

Proximity to the existing cafeterias: In order to construct a new cafeteria it mustn't be near the already established ones, so, 3 zones buffers are made around the existing cafeterias with $100,200,300 \mathrm{~m}$, so, the resulting location would be as far as possible from the them.

Proximity to classrooms: To make the resulting location as lucrative as possible it should be as close as possible to the classrooms to benefit the maximum number of students and staff, so, 3 zones buffers are made around the classrooms with $25,50,75,100,125 \mathrm{~m}$ as in Fig. 1 .

\section{RESULTS AND DISCUSSION}

Proximity to the borders: To achieve the goal of finding an optimum location for the cafeteria it has to be an open spaces in which the cafeteria will be constructed in, so, 3 zones buffers are made around the classrooms with 50 , $100,175 \mathrm{~m}$.

Erase: To prevent an overlap between the buffer zones it is necessary to separate them by using the erase tool (analysis tools---overlay---erase) where the input feature is the bigger buffer zone and the erase feature is the smaller buffer zone as in Fig. 1 and 2.

Merge: In order to combine the 3 buffer layers after conducting the erase step, Merge tool is used for that cause (geo-processing---merge) as in Fig. 3.

Polygon to raster: This step converts the polygon features (vector) to raster features knowing that he chosen value field is (buffer distance) (conversion tools to raster polygon to raster) to be able to perform the next step which deals with raster layers only as in Fig. 4.

Corresponding Author: Asma Th. Ibraheem, Department of Civil Engineering, College of Engineering, Al-Nahrain University, Baghdad, Iraq 


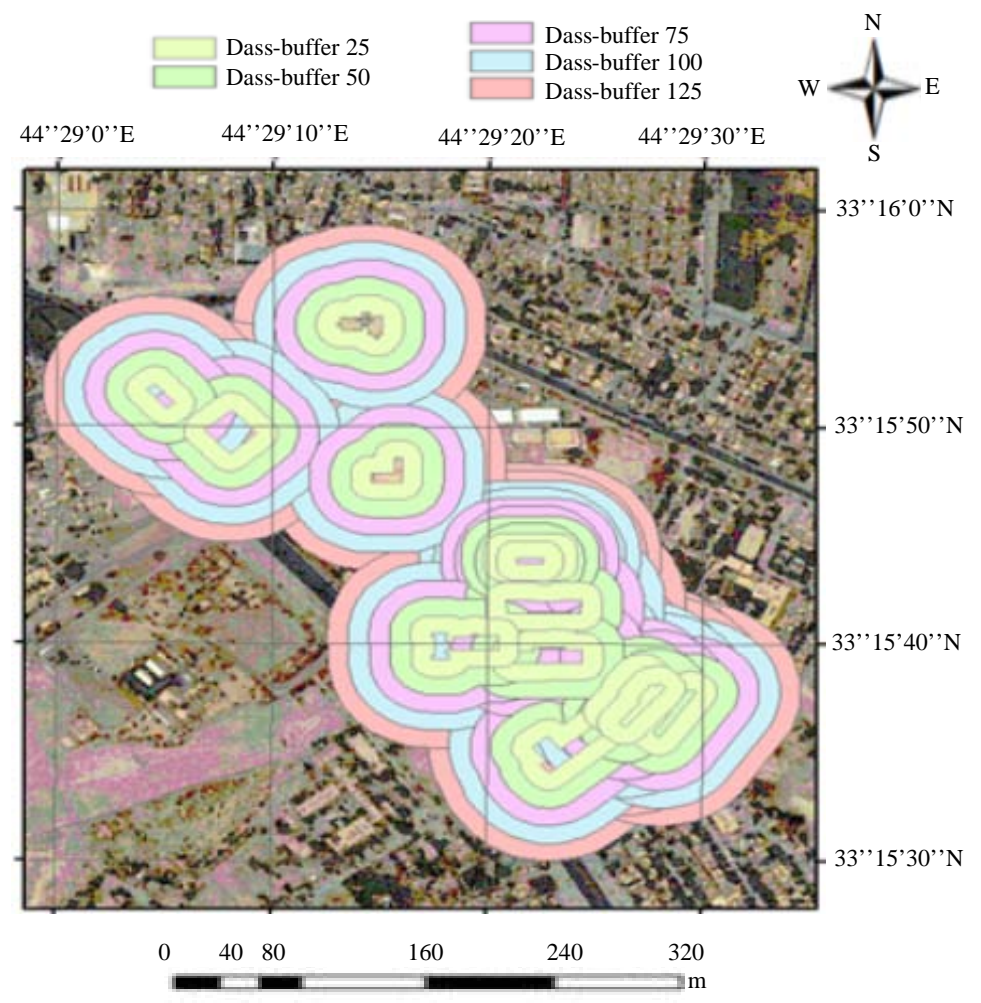

Fig. 1: Classrooms buffers
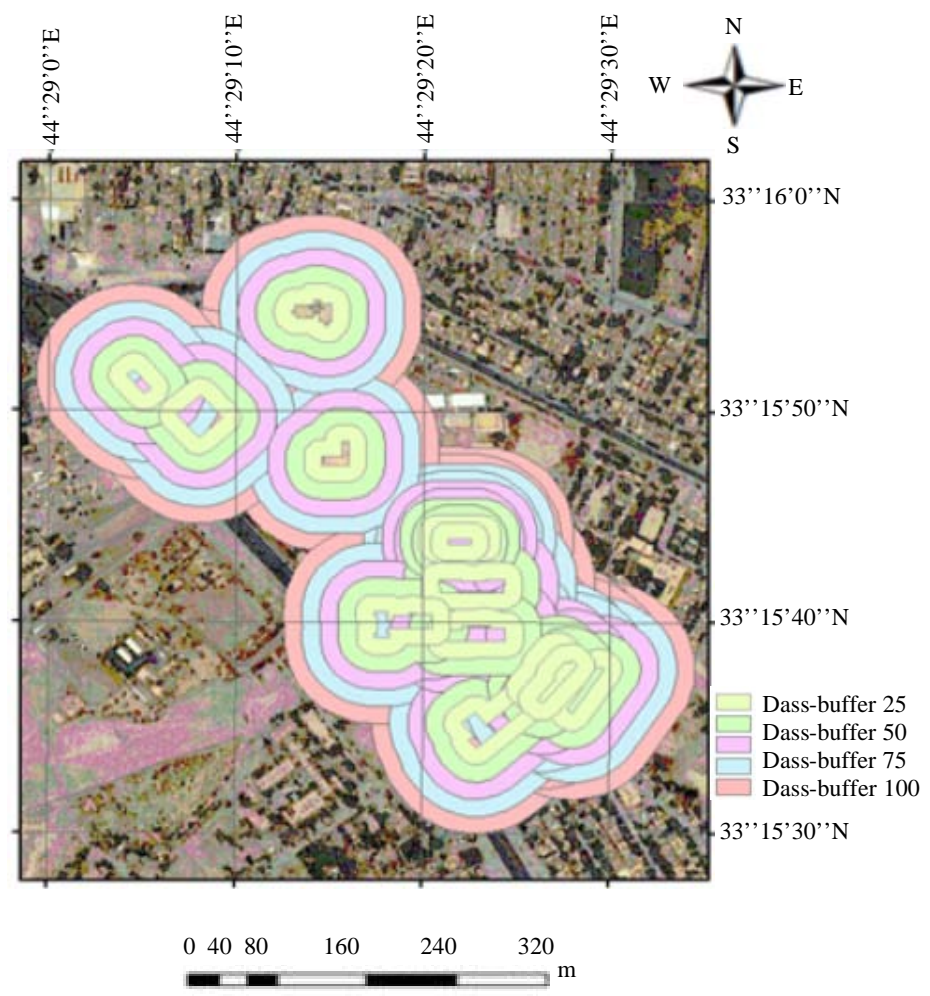

Fig. 2: Erase step for the classrooms 


\section{J. Eng. Applied Sci., 14 (3): 750-758, 2019}

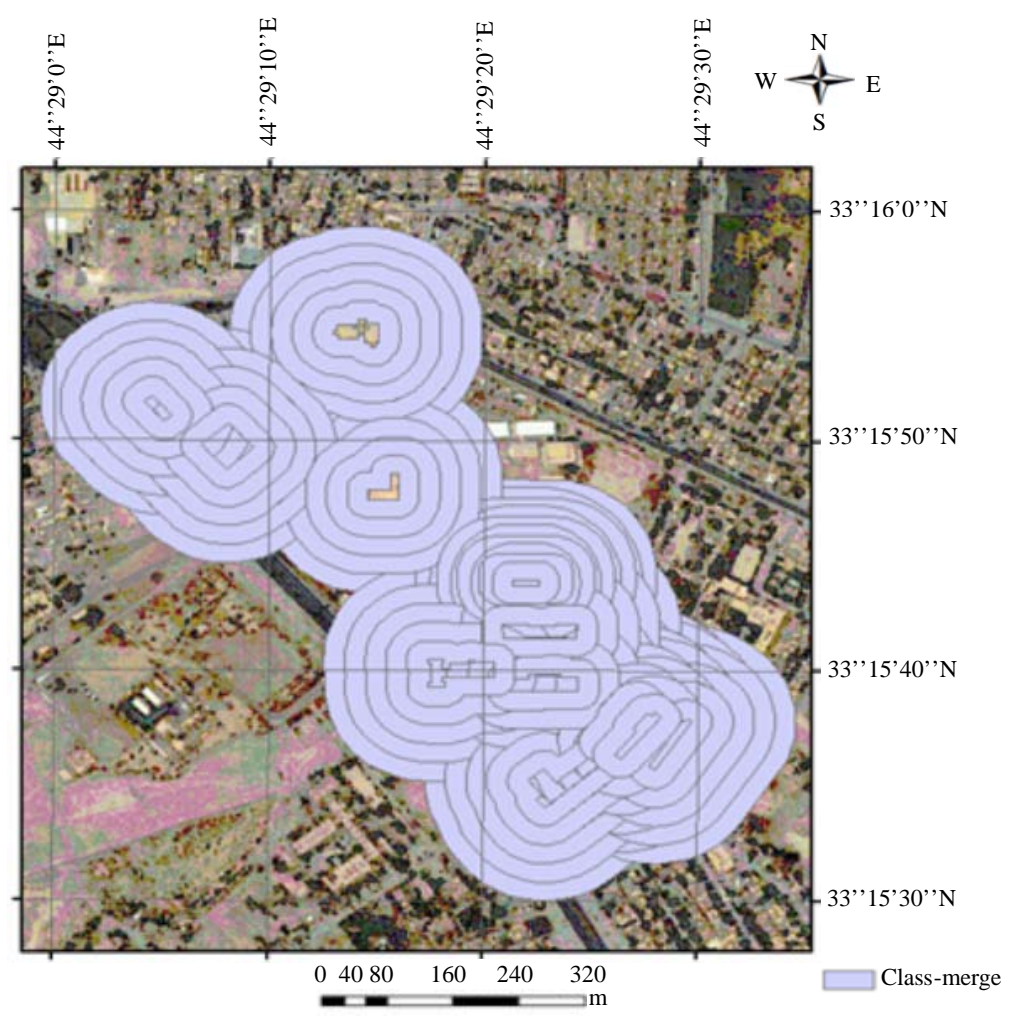

Fig. 3: Classrooms buffers merge

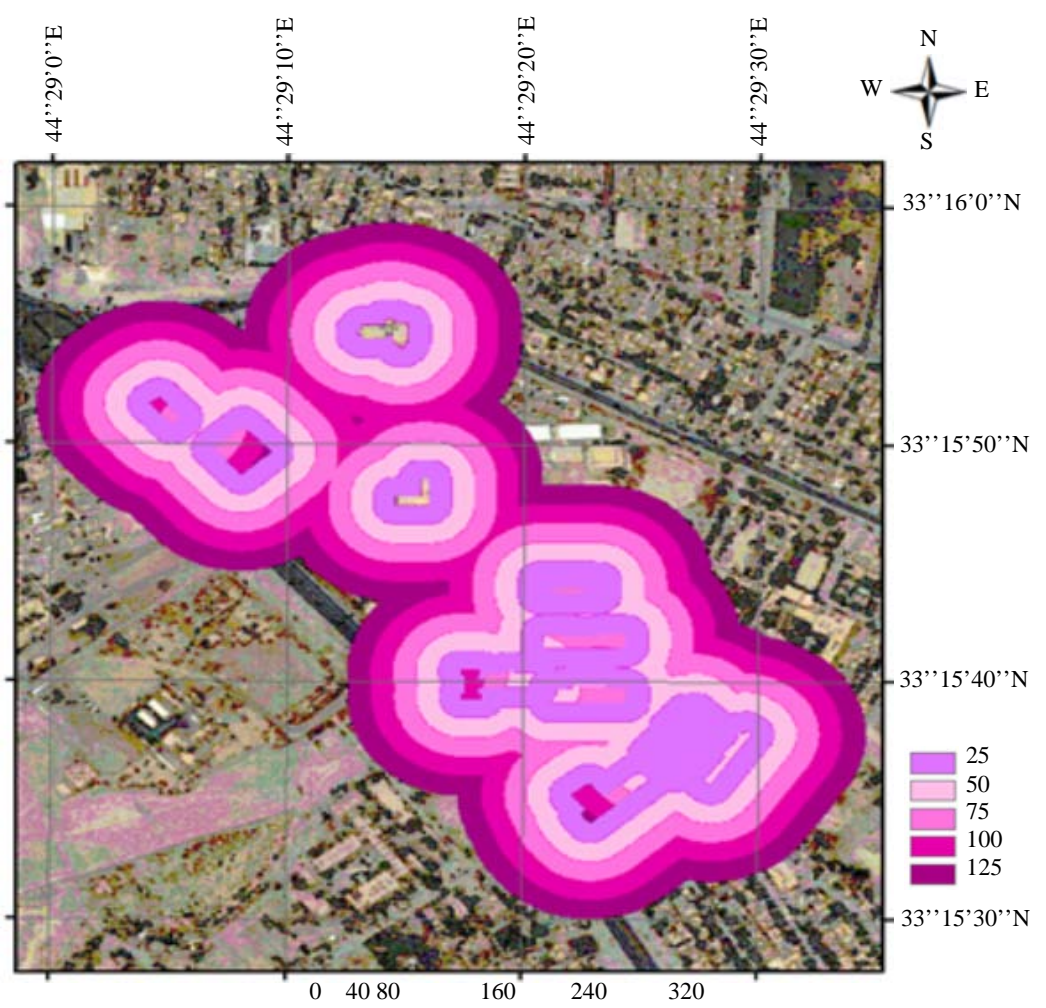

Fig. 4: Converting the polygon features (classrooms) to raster 


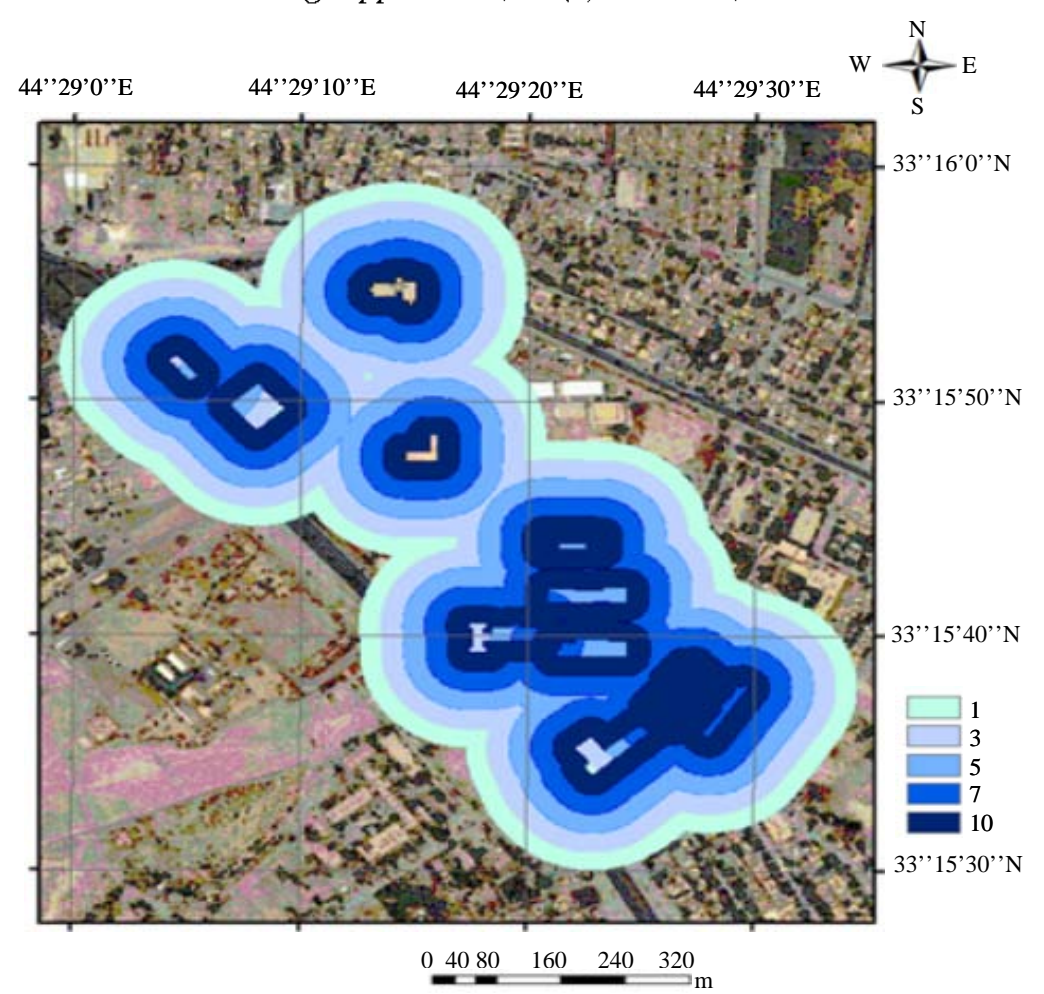

Fig. 5: Classrooms re-classification step

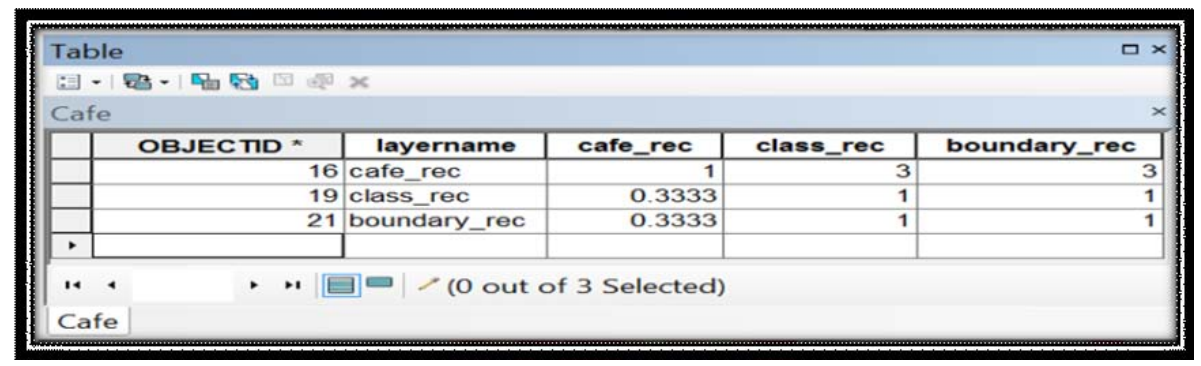

Fig. 6: AHP table for the cafe

Reclassify: This step is to change the values of the previously converted buffer zones depending on their priority meaning: (prioritizing the closest distance to the chosen feature) (Spatial analyst tools---Reclass--Reclassify) as in Fig. 5.

Finally, it comes to open the AHP extension (AHP step1) to enter the name of the geo-database that contains all the earlier work and giving a name to the newly made table then adding this table to the (Table of content) by the icon (Add data), to open in it and finding all the raster layers in that geo-database, so, any are removed and the kept layers are (reclassified classrooms, reclassified cafeterias, reclassified boundary) and self-entering the values for the comparison matrix as illustrated in Fig. 6.
Table in Fig. 6 means the (cafe) criteria is 3 times important than the reaming to criteria which have equally the same importance.

Then by opening AHP step 2 and entering the name of the table it immediately analyze the values and gives the weights of each criteria with the Consistency Index (CI), Consistency Ratio (CR) and a field showing that the matrix is consistent enough as well as in Fig. 7.

As it shows in the Fig. 7, AHP gives just weights to each criteria and there is a need to show it in a suitability map, so by the use of the tool weighted overlay this goal can be achieved by entering the resulting weights from the table (AHP-cafeCI) to get the suitability map as in Fig. 8. Figure 8 shows that the green areas are the most suitable locations and the dark red areas are the least suitable location. 


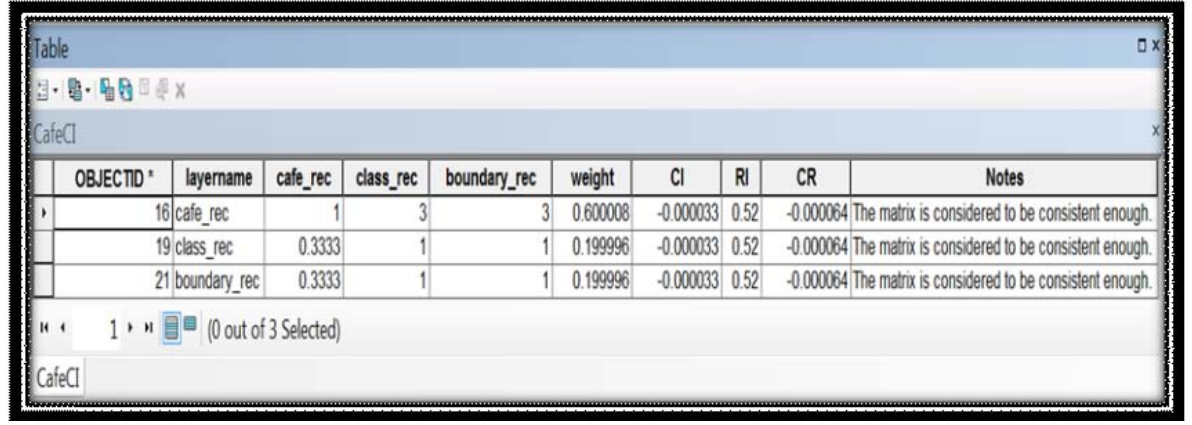

Fig. 7: AHP table cafe CI

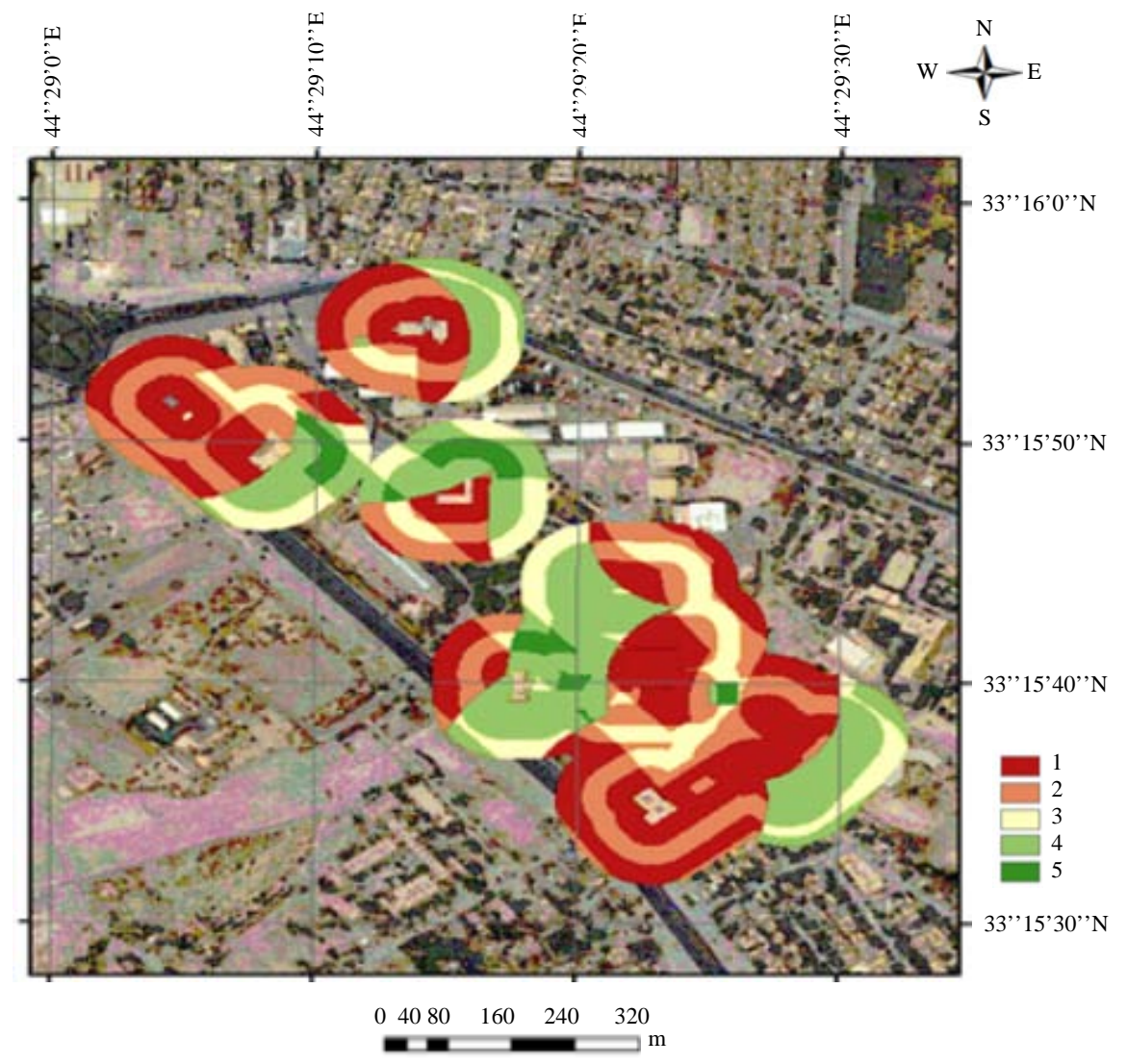

Fig. 8: Suitability map for the cafeteria

Health center: The same steps are applied for this facility and with same criteria as well resulting these tables in Fig. 9 and 10 the suitability map in Fig. 11.

Figure 11 shows that the green areas are the most suitable locations and the dark red areas are the least suitable locations.

Mosque: The same steps are applied for this facility and with same criteria as well resulting these tables in Fig. 12 and 13 and the suitability map in Fig. 14.
Figure 14 shows that the green areas are the most suitable locations and the dark red areas are the least suitable locations.

Parking: The same steps are applied for this facility and with same criteria as well, resulting these tables in Fig. 15 and 16 the suitability map in Fig. 17.

Figure 17 shows that the green areas are the most suitable locations and the dark red areas are the least suitable locations. 


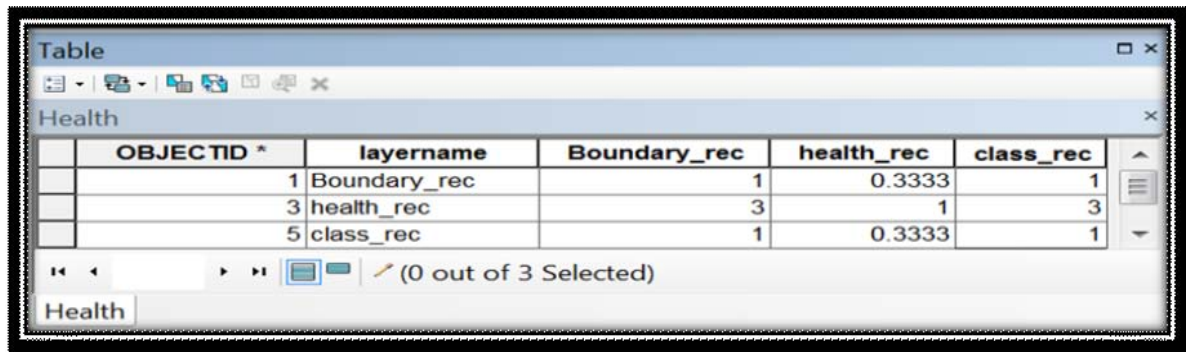

Fig. 9: AHP table for the health center

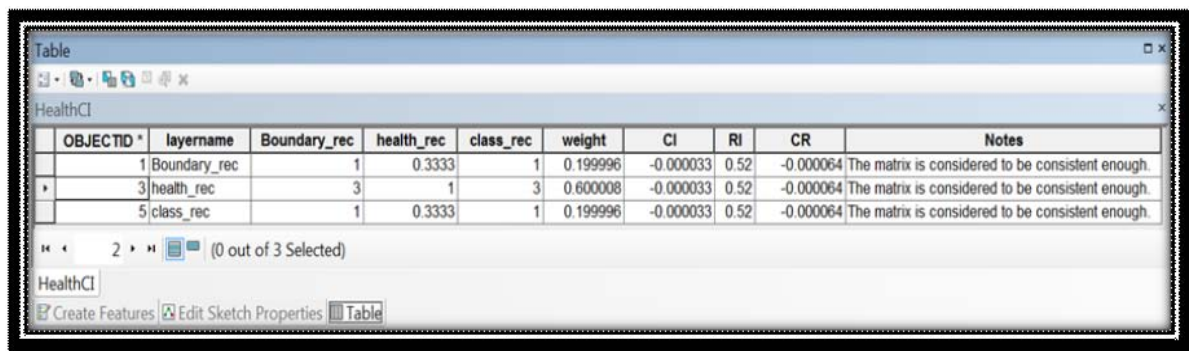

Fig. 10: AHP table health center CI

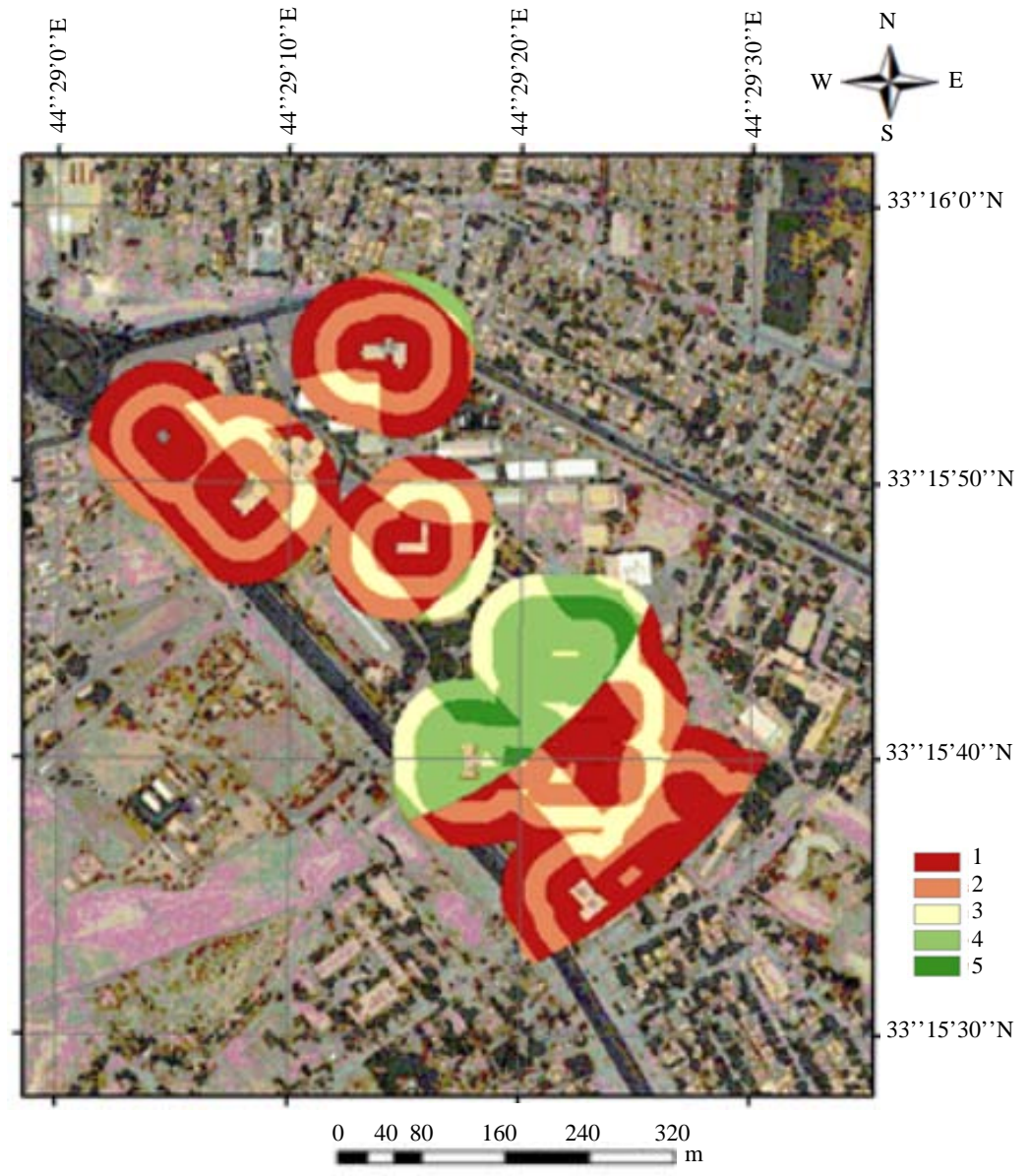

Fig. 11: Suitability map for the health center 


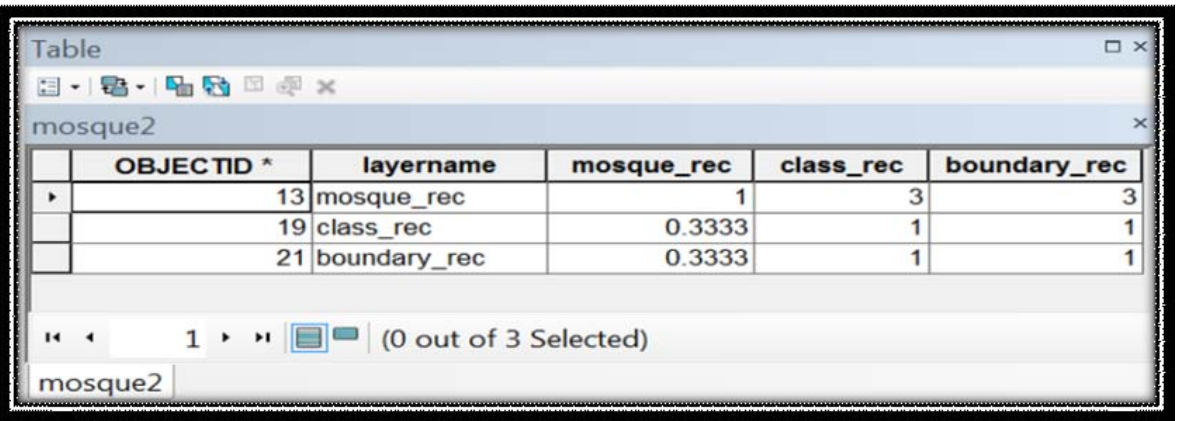

Fig. 12: AHP table for the mosque

\begin{tabular}{|c|c|c|c|c|c|c|c|c|c|c|}
\hline \multicolumn{11}{|c|}{ Table } \\
\hline \multicolumn{11}{|c|}{ 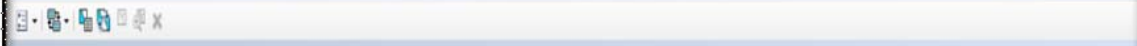 } \\
\hline \multicolumn{11}{|c|}{ mosque2C] } \\
\hline 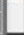 & OBJECTD: & layername & mosque_rec & class_rec & boundary_rec & weight & $\mathrm{Cl}$ & $\mathrm{Rl}$ & CR & Notes \\
\hline , & & 3 mosque_rec & 1 & 3 & 3 & 0.600008 & -0.000033 & 0.52 & .0 .00000 & The matrix is considered to be consistent enough. \\
\hline L & & 9 class_rec & 0.3333 & 1 & 1 & 0.199996 & -0.000033 & 0.52 & -0.00006 & The matrix is considered to be consistent enough. \\
\hline L & & 1 boundary_rec & 0.3333 & 1 & 1 & 0.199996 & -0.000033 & 0.52 & -0.00006 & The matrix is considered to be consistent enough. \\
\hline \multicolumn{11}{|c|}{ " . 1, " F } \\
\hline \multicolumn{11}{|c|}{ mosque2C] } \\
\hline
\end{tabular}

Fig. 13: AHP table mosque CI

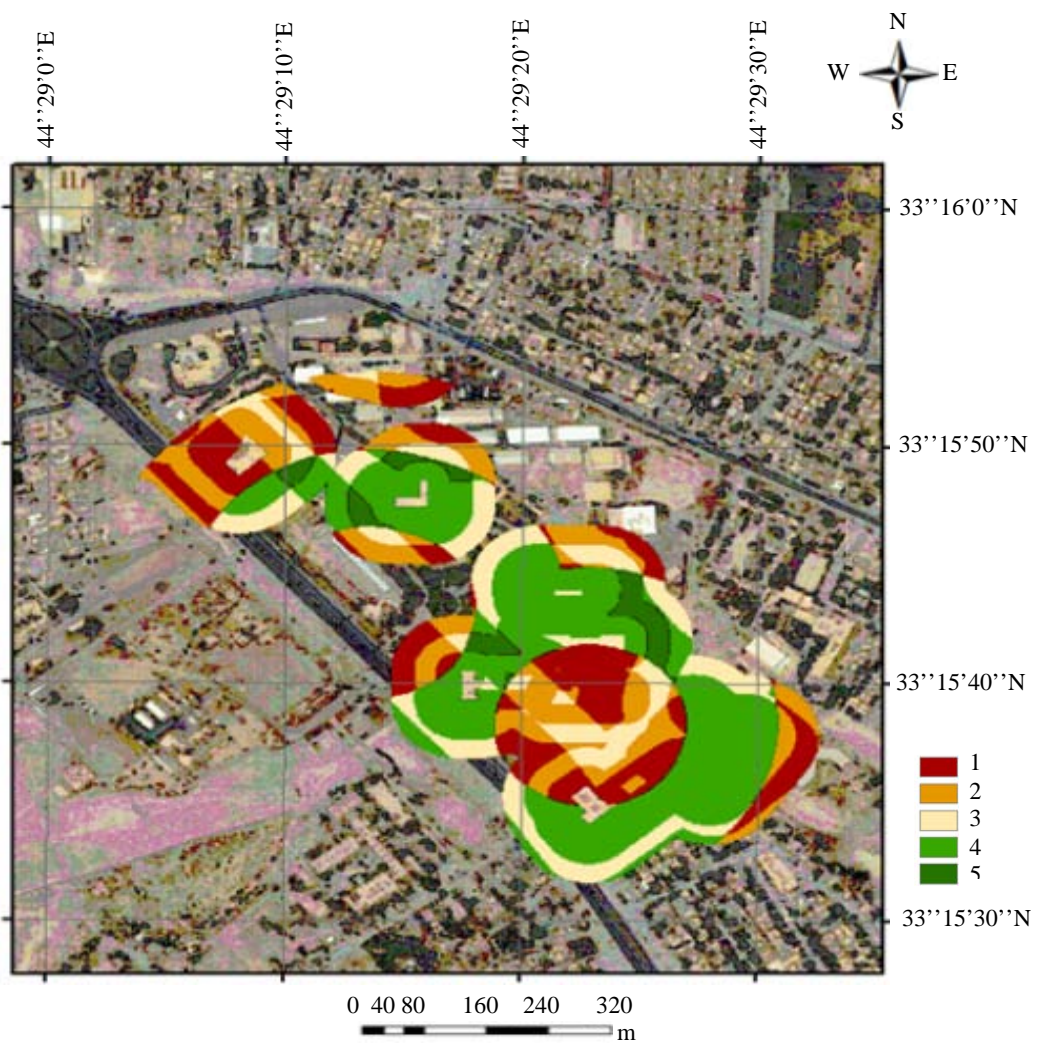

Fig. 14: Suitability map for the mosque 


\begin{tabular}{|c|c|c|c|c|c|}
\hline \multicolumn{3}{|c|}{ Table } & & & $\square \times$ \\
\hline \multicolumn{6}{|c|}{ 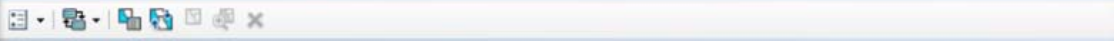 } \\
\hline \multicolumn{6}{|c|}{ Parking1 } \\
\hline & OBJECTID * & layername & park_rec & class_rec & boundary_rec \\
\hline & \multicolumn{2}{|c|}{10 park_rec } & 1 & 0.3333 & 1 \\
\hline & \multicolumn{2}{|c|}{19 class_rec } & 3 & 1 & 3 \\
\hline - & \multirow{2}{*}{\multicolumn{2}{|c|}{21 boundary_rec }} & 1 & 0.3333 & 1 \\
\hline & & & & & \\
\hline \multicolumn{6}{|c|}{ " . 21 " "圆 $/(0$ out of 3 Selected $)$} \\
\hline \multicolumn{6}{|c|}{ Parking1 } \\
\hline
\end{tabular}

Fig. 15: AHP table for the parking

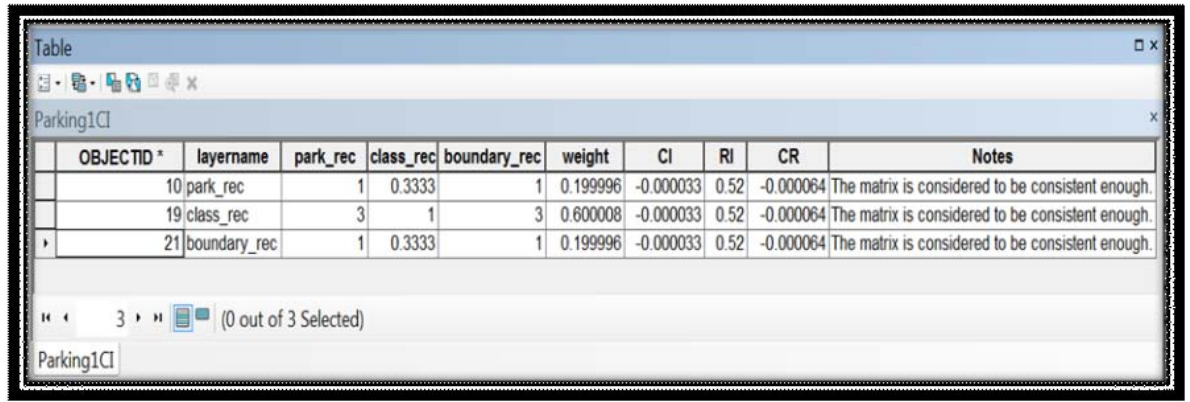

Fig. 16: AHP table parking CI

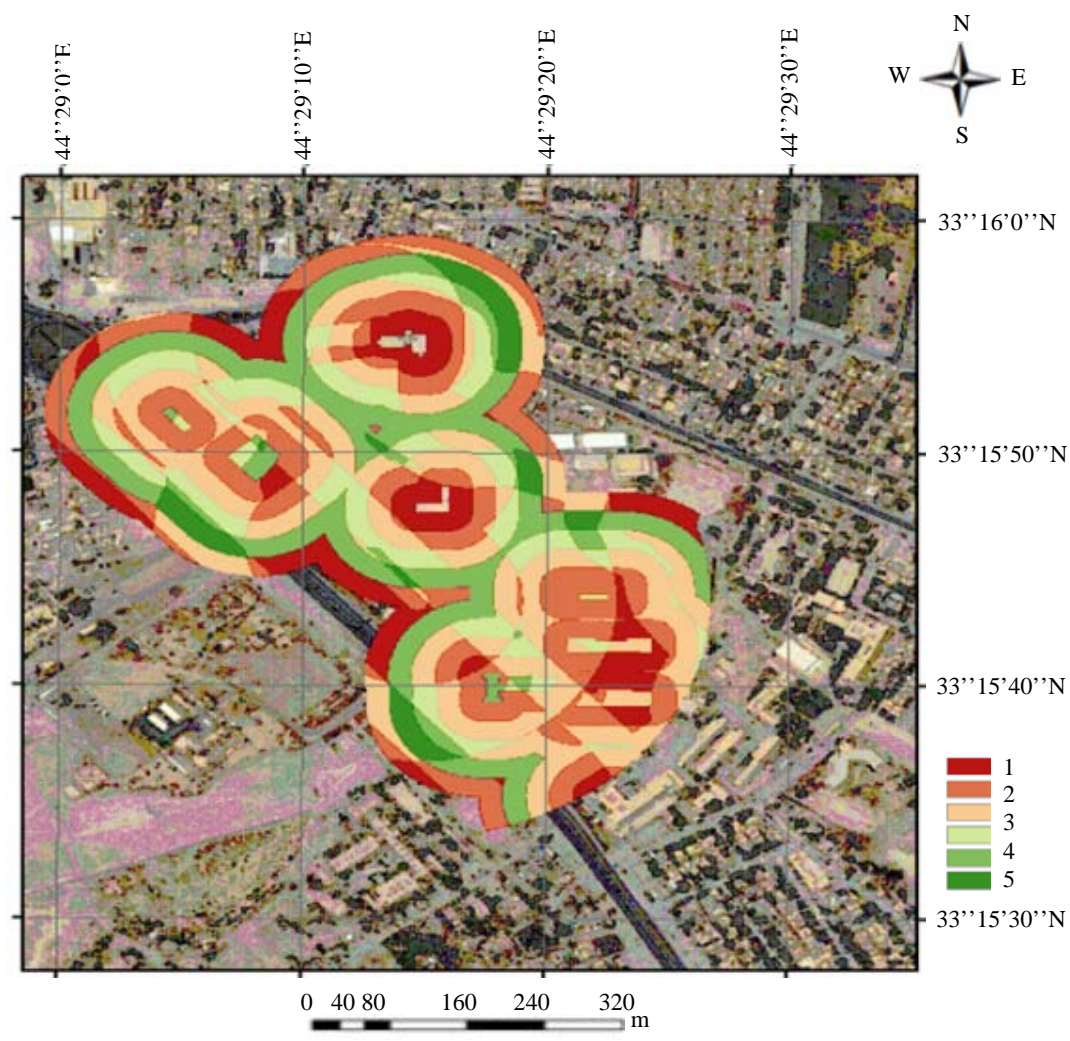

Fig. 17: Suitability map for the parking 
AHP extension calculates weight of the criteria depending on the numbers entered in the pairwise comparison matrix, also, calculates whether the matrix is consistent or not. The results were as follows: the most effective criteria concerning the cafeteria was the proximity from the existing cafeterias weight [0.6], the most effective criteria for the health center was the proximity from the exiting health center with weight [0.6] as for the mosque it was the same criteria (the proximity from the existing mosques) with weight [0.6], finally, the criteria effecting the location of the parking it was the proximity from the classrooms with weight [0.6] all of these weights were inserted in the tool (weighted overlay) to produce the suitability map for each facility.

\section{CONCLUSION}

This extension can facilitate the process of multi criteria decision making and gives a final unbiased result. Also, integrate both decision-making with digital mapping which represent a huge step forward in many sector of science such as engineering and geography etc. This extension can help to eliminate the tedious and redundant steps that were necessary to do in the field of multi criteria decision making.

\section{REFERENCES}

Akalin, M., G. Turhan and A. Sahin, 2016. The application of AHP approach for evaluating location selection elements for retail store: A case of clothing store. Intl. J. Res. Bus. Soc. Sci., 2: 1-20.

Chatterjee, D. and B. Mukherjee, 2013. Potential hospital location selection using AHP: A study in rural India. Intl. J. Comput. Appl., 71: 1-7.

Harker, P.T. and L.G. Vargas, 1987. The theory of ratio scale estimation: Saaty's analytic hierarchy process. Manag. Sci., 33: 1383-1403.

Ibraheem, A.T., A.N. Abid and A.J. Mehdi, 2015. Modern Basics and Methods for Car Parking. Lap Lambert Academic Publishing, Germany, Europe.

Kuo, R.J., S.C. Chi and S.S. Kao, 1999. A decision support system for locating convenience store through fuzzy AHP. Comput. Ind. Eng., 37: 323-326.

Mikhailov, L. and P. Tsvetinov, 2004. Evaluation of services using a fuzzy analytic hierarchy process. Applied Soft Comput., 5: 23-33. 